PROCEEDINGS OF THE

AMERICAN MATHEMATICAL SOCIETY

Volume 128, Number 8, Pages 2243-2251

S 0002-9939(99)05229-6

Article electronically published on November 29, 1999

\title{
COHEN-MACAULAYNESS OF TANGENT CONES
}

\author{
FEZA ARSLAN
}

(Communicated by Wolmer V. Vasconcelos)

\begin{abstract}
We give a criterion for checking the Cohen-Macaulayness of the tangent cone of a monomial curve by using the Gröbner basis. For a family of monomial curves, we give the full description of the defining ideal of the curve and its tangent cone at the origin. By using this family of curves and their extended versions to higher dimensions, we prove that the minimal number of generators of a Cohen-Macaulay tangent cone of a monomial curve in an affine $l$-space can be arbitrarily large for $l \geq 4$ contrary to the $l=3$ case shown by Robbiano and Valla. We also determine the Hilbert series of the associated graded ring of this family of curves and their extended versions.
\end{abstract}

\section{Introduction}

In this article, our main interest is to study the Cohen-Macaulayness of the tangent cone of a monomial curve. In general, it is important to discover whether the associated graded ring of a local ring $(R, m)$ is Cohen-Macaulay, since this property assures a better control on the blow-up of $\operatorname{Spec}(R)$ along $V(m)$; in particular it reduces the computation of the Hilbert function of the ring to a computation of the Hilbert function of an Artin local ring 11. The computation of the Hilbert function of an Artin local ring is trivial, because it has a finite number of nonzero values.

The Cohen-Macaulayness of the tangent cone of a monomial curve $C$ having parameterization

$$
x_{1}=t^{n_{1}}, x_{2}=t^{n_{2}}, \ldots, x_{l}=t^{n_{l}}
$$

can be studied both as the Cohen-Macaulayness of the associated graded ring of $A=k\left[\left[t^{n_{1}}, t^{n_{2}}, \ldots, t^{n_{l}}\right]\right]$ with respect to the maximal ideal $m=\left(t^{n_{1}}, t^{n_{2}}, \ldots, t^{n_{l}}\right)$ (which is $\bigoplus_{i=0}^{\infty} m^{i} / m^{i+1}$ and denoted by $\left.g r_{m}\left(k\left[\left[t^{n_{1}}, t^{n_{2}}, \ldots, t^{n_{l}}\right]\right]\right)\right)$ or as the CohenMacaulayness of the ring $k\left[x_{1}, x_{2}, \ldots, x_{l}\right] / I(C)_{*}$ where $I(C)$ is the defining ideal of $C$,

$$
\begin{aligned}
&\left\{f\left(x_{1}, \ldots, x_{l}\right)\right. \text { such that } f\left(x_{1}, \ldots, x_{l}\right) \in k\left[x_{1}, \ldots, x_{l}\right], f\left(t^{n_{1}}, \ldots, t^{n_{l}}\right)=0, t \\
&\text { transcendental over } k\},
\end{aligned}
$$

and $I(C)_{*}$ is the ideal generated by the polynomials $f_{*}$ for $f$ in $I(C)$, where $f_{*}$ is the homogeneous summand of $f$ of least degree.

Received by the editors May 1, 1998 and, in revised form, September 18, 1998.

1991 Mathematics Subject Classification. Primary 14H20; Secondary 13H10, 13P10.

Key words and phrases. Cohen-Macaulay ring, monomial curve, tangent cone.

The author was supported by TÜBİTAK BDP Grant. 
By using the notion of super-regular sequence, Herzog gives a necessary and sufficient condition for $\operatorname{gr}_{m}\left(k\left[\left[t^{n_{1}}, t^{n_{2}}, \ldots, t^{n_{l}}\right]\right]\right)$ to be Cohen-Macaulay [8]. In [6], Garcia obtains the same result with a different approach. Cavaliere and Niesi also attack the same problem by studying the semigroup ring $k[S]$ where $S \subset N^{2}$ is generated by $\left(n_{1}, 0\right),\left(n_{2}, n_{2}-n_{1}\right), \ldots,\left(n_{l}, n_{l}-n_{1}\right),\left(0, n_{1}\right)$. They introduce the notion of standard bases for $S$ and give a simple criterion for the Cohen-Macaulayness of the rings $k[S]$ and $g r_{m}\left(k\left[\left[t^{n_{1}}, t^{n_{2}}, \ldots, t^{n_{l}}\right]\right]\right)$ [4].

In [10, Robbiano and Valla give a characterization of standard bases, which relies on homological methods and is particularly useful while dealing with determinantal ideals. By using this theory with Herzog's [7] description of the defining ideals of monomial curves for $l=3$, they give a classification of these curves by their tangent cones at the origin. They prove that a monomial curve $C$ having parameterization

$$
x_{1}=t^{n_{1}}, x_{2}=t^{n_{2}}, x_{3}=t^{n_{3}}
$$

has Cohen-Macaulay tangent cone at the origin if and only if the minimal number of generators of the tangent cone, that is, $\mu\left(I(C)_{*}\right)$, is less than or equal to three. We investigate and show that in higher dimensions, the minimal number of generators of a Cohen-Macaulay tangent cone of a monomial curve can be arbitrarily large. Namely, in $l$-space with $l>3$, there are monomial curves with arbitrarily large $\mu\left(I(C)_{*}\right)$ and Cohen-Macaulay tangent cones.

For a family of monomial curves $C_{m}$ in 4 -space with $n_{1}=m(m+1), n_{2}=$ $m(m+1)+1, n_{3}=(m+1)^{2}$ and $n_{4}=(m+1)^{2}+1,(m \geq 2)$, we give a description of the defining ideal $I\left(C_{m}\right)$ (Proposition 3.2$)$ and by using Gröbner bases, we compute a minimal generator set for $I\left(C_{m}\right)_{*}$ (Proposition 3.4) such that $\mu\left(I\left(C_{m}\right)_{*}\right)=2 m+2$ and show that $k\left[x_{1}, x_{2}, x_{3}, x_{4}\right] / I\left(C_{m}\right)_{*}$ is Cohen-Macaulay (Theorem 3.1) by using the checking criterion given in Section 2 (Theorem 2.1). We extend this result to higher dimensions. We also determine the Hilbert series of the associated graded ring of this family of curves and their extended versions (Remark 3.7 and Remark 4.4).

Let us summarize the notation: $C$ will denote a curve in $l$-affine space, having parameterization

$$
x_{1}=t^{n_{1}}, x_{2}=t^{n_{2}}, \ldots, x_{l}=t^{n_{l}}
$$

where $n_{1}, n_{2}, \ldots, n_{l}$ are positive integers with $1<n_{1}<n_{2}<\ldots<n_{l}$ and $n_{1}, n_{2}, \ldots, n_{l}$ is a minimal set of generators for the numerical semigroup $\left\langle n_{1}, n_{2}, \ldots, n_{l}\right\rangle=\{n \mid n=$ $\sum_{i=1}^{l} a_{i} n_{i}, a_{i}$ 's are nonnegative integers $\} . I(C)$ is the defining ideal of $C . I(C)_{*}$ is the ideal generated by the polynomials $f_{*}$ for $f$ in $I(C)$, where $f_{*}$ is the homogeneous summand of $f$ of least degree, and $\mu\left(I(C)_{*}\right)$ is the minimal number of generators of the tangent cone of the monomial curve $C$. We denote the associated graded ring of $A=k\left[\left[t^{n_{1}}, t^{n_{2}}, \ldots, t^{n_{l}}\right]\right]$ with respect to the maximal ideal $m=\left(t^{n_{1}}, t^{n_{2}}, \ldots, t^{n_{l}}\right)$ by $g r_{m}\left(k\left[\left[t^{n_{1}}, t^{n_{2}}, \ldots, t^{n_{l}}\right]\right]\right)$.

\section{When IS $g r_{m}\left(k\left[\left[t^{n_{1}}, t^{n_{2}}, \ldots, t^{n_{l}}\right]\right]\right)$ Cohen-Macaulay?}

In this section, we state and prove the following theorem, which we use for checking the Cohen-Macaulayness of the tangent cone of a monomial curve $C$ by considering the ideal $I(C)_{*}$

Theorem 2.1. Let $C$ be a curve as in (1.2). Let $g_{1}, \ldots, g_{s}$ be a minimal Gröbner basis for $I(C)_{*}$ with respect to a reverse lexicographic order that makes $x_{1}$ the lowest 
variable. Then $\operatorname{gr}_{m}\left(k\left[\left[t^{n_{1}}, t^{n_{2}}, \ldots, t^{n_{l}}\right]\right]\right)$ is Cohen-Macaulay if and only if $x_{1} \chi \operatorname{in}\left(g_{i}\right)$ for $1 \leq i \leq s$, where in $\left(g_{i}\right)$ is the leading term of $g_{i}$.

To prove this theorem, we first recall the following definition and lemmas. (Here, we only give the definition of the reverse lexicographic order. For definitions of monomial order, multidegree, Gröbner basis, etc., see [5].)

Definition 2.2 ([5, p. 57] Reverse Lex Order). Let $\alpha, \beta \in Z_{\geq 0}^{l}$. We say $\alpha>_{\text {grevlex }}$ $\beta$ if

$$
|\alpha|=\sum_{i=1}^{n} \alpha_{i}>|\beta|=\sum_{i=1}^{n} \beta_{i} \text {, or }|\alpha|=|\beta|
$$

in $\alpha-\beta \in Z^{l}$, the right-most nonzero entry is negative.

Lemma 2.3 (Bayer-Stillmann [12, p. 32]). Let $I \subset k\left[x_{1}, \ldots, x_{l}\right]$ be a homogeneous ideal and consider reverse lexicographic order that makes $x_{1}$ the lowest variable. Then

$$
I: x_{1}=I \Leftrightarrow \operatorname{in}(I): x_{1}=\operatorname{in}(I)
$$

where in $(I)$ is the ideal generated by in $(f)$ 's with $f \in I$.

Lemma 2.4 (6, Theorem 7]). $g r_{m}\left(k\left[\left[t^{n_{1}}, t^{n_{2}}, \ldots, t^{n_{l}}\right]\right]\right)$ is Cohen-Macaulay if and only if $t^{n_{1}}$ is not a zero divisor in $g_{m}\left(k\left[\left[t^{n_{1}}, t^{n_{2}}, \ldots, t^{n_{l}}\right]\right]\right)$.

Proof of Theorem 2.1. From the isomorphism

$$
g r_{m}\left(k\left[\left[t^{n_{1}}, t^{n_{2}}, \ldots, t^{n_{l}}\right]\right]\right) \cong k\left[x_{1}, x_{2}, \ldots, x_{l}\right] / I(C)_{*}
$$

$t^{n_{1}}$ is not a zero divisor in $g r_{m}\left(k\left[\left[t^{n_{1}}, t^{n_{2}}, \ldots, t^{n_{l}}\right]\right]\right)$ if and only if $x_{1}$ is not a zero divisor in $k\left[x_{1}, x_{2}, \ldots, x_{l}\right] / I(C)_{*}$. Combining this with Lemma 2.3 and Lemma 4, $g r_{m}\left(k\left[\left[t^{n_{1}}, t^{n_{2}}, \ldots, t^{n_{l}}\right]\right]\right)$ is Cohen-Macaulay $\Leftrightarrow I(C)_{*}: x_{1}=I(C)_{*} \Leftrightarrow i n\left(I(C)_{*}\right)$ : $x_{1}=i n\left(I(C)_{*}\right)$ with respect to the reverse lexicographic order that makes $x_{1}$ the lowest variable. From the definition of a minimal Gröbner basis,

$$
\operatorname{in}\left(I(C)_{*}\right)=\left(i n\left(g_{1}\right), \ldots, i n\left(g_{s}\right)\right) \text { and } i n\left(g_{i}\right) \chi i n\left(g_{j}\right) \text { if } i \neq j .
$$

Thus, $g r_{m}\left(k\left[\left[t^{n_{1}}, t^{n_{2}}, \ldots, t^{n_{l}}\right]\right]\right)$ is Cohen-Macaulay if and only if $x_{1}$ does not divide $\operatorname{in}\left(g_{i}\right)$ for $1 \leq i \leq s$.

\section{A family of MONOMial CURVES IN 4-SPACE WHICH HAVE CM TANGENT CONES}

In this section, we check the Cohen-Macaulayness of the tangent cone of the monomial curves $C_{m}$ having the parameterization

$$
x_{1}=t^{m(m+1)}, x_{2}=t^{m(m+1)+1}, x_{3}=t^{(m+1)^{2}}, x_{4}=t^{(m+1)^{2}+1}
$$

with $m \geq 2$. Our main result is the following theorem, which we prove in the end of this section.

Theorem 3.1. The monomial curve $C_{m}$ having parameterization as in (3.1) has Cohen-Macaulay tangent cone at the origin.

Our first aim is to give a complete description of the defining ideal $I\left(C_{m}\right)$. From our computations in Macaulay [2] with particular values for $m$, we formulate a set of generators and prove that the set formulated is indeed a generator set for $I\left(C_{m}\right)$ by applying the method Bresinsky used in [3] which depends on work of Herzog on semigroups [7]. 
Proposition 3.2. $I\left(C_{m}\right)$ is generated by $G=\left\{g_{i}=x_{1}^{m-i} x_{3}^{i+1}-x_{2}^{m-i+1} x_{4}^{i}\right.$ with $0 \leq i \leq m, f_{j}=x_{3}^{j} x_{4}^{m-j}-x_{1}^{j+1} x_{2}^{m-j}$ with $0 \leq j \leq m$ and $\left.h=x_{1} x_{4}-x_{2} x_{3}\right\}$.

From [7] Proposition 1.4], $I\left(C_{m}\right)$ is generated by binomials $F(\nu, \mu)$ of the form

$$
F(\nu, \mu)=x_{1}^{\nu_{1}} \ldots x_{4}^{\nu_{4}}-x_{1}^{\mu_{1}} \ldots x_{4}^{\mu_{4}}, \quad \partial(F(\nu, \mu))=\sum_{i=1}^{4} \nu_{i} n_{i}=\sum_{i=1}^{4} \mu_{i} n_{i}
$$

with $\nu_{i}$ or $\mu_{i}=0,1 \leq i \leq l$, and $n_{1}=m(m+1), n_{2}=m(m+1)+1, n_{3}=$ $(m+1)^{2}, n_{4}=(m+1)^{2}+1$.

Thus, we can prove the lemma by showing that, for all $F(\nu, \mu)$, there is an element $f \in\left(f_{0}, f_{1}, \ldots, f_{m}, g_{0}, g_{1}, \ldots, g_{m}, h\right)$ such that $F(\nu, \mu)-f=\prod_{i=1}^{4} x_{i}^{a_{i}} g$ with $g=0$ or $g=F\left(\nu^{\prime}, \mu^{\prime}\right)$ with $\partial\left(F\left(\nu^{\prime}, \mu^{\prime}\right)\right)<\partial(F(\nu, \mu))$, since this proves that any binomial $F(\nu, \mu)$ can be generated by $\left\{f_{0}, f_{1}, \ldots, f_{m}, g_{0}, g_{1}, \ldots, g_{m}, h\right\}$.

Thus, the following lemma is crucial for our purpose, since it determines the polynomials $x_{i_{1}}^{\nu_{i_{1}}}-x_{i_{2}}^{\nu_{i_{2}}} x_{i_{3}}^{\nu_{i_{3}}} x_{i_{4}}^{\nu_{i_{4}}}$ in $I\left(C_{m}\right)$ with $1 \leq i_{1}, i_{2}, i_{3}, i_{4} \leq 4$ and $\nu_{i_{1}}$ minimal.

Lemma 3.3. Let $n_{1}=m(m+1), n_{2}=m(m+1)+1, n_{3}=(m+1)^{2}, n_{4}=(m+1)^{2}+1$ with $m \geq 2$. If $\nu_{i_{1}} n_{i_{1}} \in\left\langle n_{i_{2}}, n_{i_{3}}, n_{i_{4}}\right\rangle$, with $1 \leq i_{1}, i_{2}, i_{3}, i_{4} \leq 4$ (all $i_{k}$ 's are distinct), $\nu_{i_{1}}$ strictly positive and minimal, then $\nu_{1}=m+1, \nu_{2}=m+1, \nu_{3}=m$, $\nu_{4}=m$.

Proof. For $i_{1}=1$, we have the equation

$$
\nu_{1} m(m+1)=\mu_{2}(m(m+1)+1)+\mu_{3}(m+1)^{2}+\mu_{4}\left((m+1)^{2}+1\right)
$$

and $m+1 \mid \mu_{2}+\mu_{4}$ follows immediately. Thus, if either $\mu_{2}$ or $\mu_{4} \neq 0$, then $\mu_{2}+\mu_{4} \geq m+1$. Also, from (3.3), $\nu_{1}>\mu_{2}+\mu_{3}+\mu_{4}$ and substituting $\mu_{2}+\mu_{4} \geq m+1$ in this inequality, we obtain $\nu_{1}>m+1$. If $\mu_{2}=\mu_{4}=0$, then $\mu_{3}=m$ and $\nu_{1}=m+1$. Thus, the minimal positive value for $\nu_{1}$ is $m+1$.

For $i_{1}=2$, we have the equation

$$
\nu_{2}(m(m+1)+1)=\mu_{1} m(m+1)+\mu_{3}(m+1)^{2}+\mu_{4}\left((m+1)^{2}+1\right),
$$

from which $\mu_{4}$ and $m+1 \mid \nu_{2}-\mu_{4}$ follow. Thus, $\nu_{2} \geq m+1$. Since $\nu_{2}=m+1$, $\mu_{1}=m, \mu_{3}=1$ and $\mu_{4}=0$ satisfy the equation (3.4), the minimal positive value for $\nu_{2}$ is $m+1$.

For $i_{1}=3$, we have the equation

$$
\nu_{3}(m+1)^{2}=\mu_{1} m(m+1)+\mu_{2}(m(m+1)+1)+\mu_{4}\left((m+1)^{2}+1\right)
$$

and $m+1 \mid \mu_{2}+\mu_{4}$ follows immediately. If either $\mu_{2}$ or $\mu_{4} \neq 0$, then $\mu_{2}+\mu_{4} \geq m+1$. Thus,

$$
\begin{aligned}
& \nu_{3}(m+1)^{2} \geq \mu_{2}(m(m+1)+1)+\mu_{4}\left((m+1)^{2}+1\right), \\
& \nu_{3}(m+1)^{2} \geq\left(\mu_{2}+\mu_{4}\right)(m(m+1)+1), \\
& \nu_{3}(m+1)^{2} \geq(m+1)(m(m+1)+1),
\end{aligned}
$$

from which we obtain $\nu_{3}>m$. If $\mu_{2}=\mu_{4}=0$, then $\nu_{3}=m$ and $\mu_{1}=m+1$. Thus, the minimal positive value for $\nu_{3}$ is $m$.

For $i_{1}=4$, we have the equation

$$
\nu_{4}\left((m+1)^{2}+1\right)=\mu_{1} m(m+1)+\mu_{2}(m(m+1)+1)+\mu_{3}(m+1)^{2} .
$$

If $\nu_{4}>\mu_{2}$, then $m+1 \mid \nu_{4}-\mu_{2}$ and $\nu_{4} \geq m+1$. If $\nu_{4}=\mu_{2}$, then $\nu_{4}=\mu_{1} m+\mu_{3}(m+1)$ and $\nu_{4} \geq m$. Otherwise, if $\nu_{4}<\mu_{2}$, then by substituting $\mu_{2}=\nu_{4}+i$ with $i>0$, we have 


$$
\nu_{4}(m+1)=\mu_{1} m(m+1)+i(m(m+1)+1)+\mu_{3}(m+1)^{2}
$$

and $\nu_{4}>m$. Since $\nu_{4}=m, \mu_{1}=1, \mu_{2}=m$ and $\mu_{3}=0$ satisfy the equation (3.6), the minimal positive value for $\nu_{4}$ is $m$.

Observing that Lemma 3.3 gives the polynomials $g_{0}, f_{0}$ and $f_{m}$ in $G$, we can now prove Proposition 3.2 .

Proof of Proposition 3.2. For any $F(\nu, \mu)$, if $\nu_{4}=\mu_{4}=0$, then $F(\nu, \mu) \in I\left(C_{m}\right) \cap$ $k\left[x_{1}, x_{2}, x_{3}\right]$. Since $\left\langle m(m+1), m(m+1)+1,(m+1)^{2}\right\rangle$ is symmetric, $I\left(C_{m}\right) \cap$ $k\left[x_{1}, x_{2}, x_{3}\right]=\left(g_{0}, f_{m}\right) \subset\left(f_{0}, f_{1}, \ldots, f_{m}, g_{0}, g_{1}, \ldots, g_{m}, h\right)$ from [7]. Thus, let us consider the binomials $F(\nu, \mu)$ with $\nu_{4} \neq 0$ :

1. If exactly one $\nu_{i}=0$ : i) $\nu_{1}=0$; then $f=x_{1}^{\mu_{1}-(m+1)} f_{m}$, ii) $\nu_{2}=0$; then $f=x_{2}^{\mu_{2}-(m+1)} g_{0}$, iii) $\nu_{3}=0$; then $f=-x_{3}^{\mu_{3}-m} f_{m}$.

2. $\nu_{1}=\nu_{2}=\nu_{3}=0$; then $\nu_{4} \geq m$, i) $\mu_{1}=\mu_{2}=0$; then $\mu_{3} \geq m$ and $f=x_{4}^{\nu_{4}-m} f_{0}-x_{3}^{\mu_{3}-m} f_{m}$, ii) $\mu_{1}$ or $\mu_{2} \neq 0$; then $f=x_{4}^{\nu_{4}-m} f_{0}$.

3. i) $\nu_{2}=\nu_{3}=0, \nu_{1} \neq 0$; then $f=x_{1}^{\nu_{1}-1} x_{4}^{\nu_{4}-1} h$.

ii) $\nu_{1}=\nu_{2}=0, \nu_{3} \neq 0$ : If $\mu_{1}=0$, then $f=x_{2}^{\mu_{2}-(m+1)} g_{0}$. Otherwise, if $\nu_{4} \geq m$, we have $f=x_{3}^{\nu_{3}} x_{4}^{\nu_{4}-m} f_{0}$, and if $\nu_{3} \geq m$, we have $f=x_{3}^{\nu_{3}-m} x_{4}^{\nu_{4}} f_{m}$. The only remaining case is $\nu_{4}, \nu_{3}<m$. Assume that $\nu_{4}<\mu_{2}$. With this assumption, the equation

$$
\nu_{3}(m+1)^{2}+\nu_{4}\left((m+1)^{2}+1\right)=\mu_{1} m(m+1)+\mu_{2}(m(m+1)+1)
$$

gives $\mu_{2}=\nu_{4}+k(m+1)$ where $k \geq 1$. Substituting this in the equation (3.7) and simplifying, we obtain

$$
\nu_{3}(m+1)+\nu_{4}=\mu_{1} m+k(m(m+1)+1) .
$$

But this equation gives

$$
\begin{aligned}
\nu_{3}+\nu_{4} & =\mu_{1} m+k(m(m+1)+1)-\nu_{3} m \\
& >m+(m(m+1)+1)-(m-1) m>2 m-2
\end{aligned}
$$

which is a contradiction since $\nu_{3}, \nu_{4}<m$. Thus, $\nu_{4} \geq \mu_{2}$. From equation (3.7), $(m+1) \mid \nu_{4}-\mu_{2}$ so that $\nu_{4}=\mu_{2}$. Substituting $\nu_{4}=\mu_{2}$ in equation (3.7), we obtain

$$
\mu_{1} m-\nu_{3} m=\nu_{3}+\nu_{4}
$$

which gives $m \mid \nu_{3}+\nu_{4}$. Thus, $f=f_{j}$ for some $j$ with $1 \leq j \leq m-1$.

iii) $\nu_{1}=\nu_{3}=0, \nu_{2} \neq 0$ a) If $\nu_{4} \geq m$, then there are two cases: If $\mu_{1} \neq 0$, $f=x_{4}^{\nu_{4}-m} x_{2}^{\nu_{2}} f_{0}$. If $\mu_{1}=0$, then $\mu_{3} \geq m$ and $f=-x_{3}^{\nu_{3}-(m+1)}\left(x_{3} f_{m}+x_{1} g_{0}\right)$. b) If $\nu_{2} \geq m+1$, then $f=-x_{4}^{\nu_{4}} x_{2}^{\nu_{2}-(m+1)} g_{0}$. c) If $\nu_{4}<m, \nu_{2}<m+1$, then from the equation

$$
\begin{gathered}
\nu_{2}((m+1) m+1)+\nu_{4}\left((m+1)^{2}+1\right)=\nu_{1} m(m+1)+\nu_{3}(m+1)^{2} \\
m+1 \mid \nu_{2}+\nu_{4} \text { and } \nu_{2}+\nu_{4}=m+1 . \text { Thus, } f=g_{i} \text { for some } i \text { with } 1 \leq i \leq m-1 .
\end{gathered}
$$

Knowing the description of the ideal $I\left(C_{m}\right)$, it is possible to compute a set of generators of $I\left(C_{m}\right)_{*}$ by using the following algorithm. (The standard reference for material used related to Gröbner theory is [5].) We first find a generator set of $I\left(C_{m}\right)^{h} \subset k\left[t, x_{1}, x_{2}, x_{3}, x_{4}\right]$ which is the homogenization of $I\left(C_{m}\right)$. It can be 
found by homogenizing the elements of a Gröbner basis of $I\left(C_{m}\right)$ with respect to any graded monomial order by using the homogenization variable $t$. From the obtained generator set of $I\left(C_{m}\right)^{h}$, another Gröbner basis $G_{1}, \ldots, G_{s}$ is computed with respect to a monomial order, such that among monomials of the same total degree, any monomial involving $t$ is greater than any monomial involving only $x_{1}, \ldots, x_{4}$. Then $I\left(C_{m}\right)_{*}$ is generated by the homogeneous summands of the least degree of $G_{1}\left(1, x_{1}, . ., x_{4}\right), \ldots, G_{s}\left(1, x_{1}, \ldots, x_{4}\right)$.

Proposition 3.4. $I\left(C_{m}\right)_{*}$ is generated by $G_{*}=\left\{g_{i}=x_{1}^{m-i} x_{3}^{i+1}-x_{2}^{m-i+1} x_{4}^{i}\right.$ with $0 \leq i \leq m-1, f_{j}^{\prime}=x_{3}^{j} x_{4}^{m-j}$ with $\left.0 \leq j \leq m, h=x_{1} x_{4}-x_{2} x_{3}\right\}$.

The proof is a direct application of the tangent cone algorithm with the following lemmas.

Lemma 3.5. $G=\left\{g_{i}=x_{1}^{m-i} x_{3}^{i+1}-x_{2}^{m-i+1} x_{4}^{i}\right.$ with $0 \leq i \leq m, f_{j}=x_{3}^{j} x_{4}^{m-j}-$ $x_{1}^{j+1} x_{2}^{m-j}$ with $\left.0 \leq j \leq m, h=x_{1} x_{4}-x_{2} x_{3}\right\}$ is a Gröbner basis with respect to the graded lexicographic order with $x_{4}>x_{2}>x_{3}>x_{1}$.

Proof. For $i<j$,

$$
\begin{aligned}
S\left(g_{i}, g_{j}\right) & =x_{4}^{j-i} x_{3}^{i+1} x_{1}^{m-i}-x_{2}^{j-i} x_{1}^{m-j} x_{3}^{j+1} \\
& =x_{1}^{m-j} x_{3}^{i+1}\left(x_{1}^{j-i} x_{4}^{j-i}-x_{2}^{j-i} x_{3}^{j-i}\right)=\left(x_{4} x_{1}-x_{2} x_{3}\right) p_{1}
\end{aligned}
$$

which shows that $S\left(g_{i}, g_{j}\right) \rightarrow_{G} 0 . \quad S\left(g_{i}, h\right)=x_{1}^{m-i+1} x_{3}^{i+1}-x_{2}^{m-i+2} x_{4}^{i-1} x_{3}=$ $x_{3} g_{i-1}$, so that $S\left(g_{i}, h\right) \rightarrow_{G} 0$. Also, $S\left(f_{i}, f_{j}\right)=x_{1}^{j-i} x_{3}^{i} x_{4}^{m-i}-x_{2}^{j-i} x_{3}^{j} x_{4}^{m-j}=$ $x_{3}^{i} x_{4}^{m-j}\left(x_{1}^{j-i} x_{4}^{j-i}-x_{2}^{j-i} x_{3}^{j-i}\right)=\left(x_{4} x_{1}-x_{2} x_{3}\right) p_{2}$. Thus, $S\left(f_{i}, f_{j}\right) \rightarrow_{G} 0 . S\left(f_{i}, h\right)=$ $x_{3}^{i} x_{4}^{m-i+1}-x_{2}^{m-i+1} x_{1}^{i} x_{3}=x_{3} f_{i-1}$, and $S\left(f_{i}, h\right) \rightarrow_{G} 0$. For $i<j, S\left(f_{i}, g_{j}\right)=$ $x_{3}^{i+1} x_{1}^{j-i} f_{m}-x_{3}^{j} g_{m-j+i}$ which shows that $S\left(f_{i}, g_{j}\right) \rightarrow_{G} 0$, and the case $i \geq j$ is similar.

This lemma gives us the opportunity to obtain $I\left(C_{m}\right)^{h}$ by homogenizing the generators of $G$ so that $I\left(C_{m}\right)^{h}$ is generated by

$G^{h}=\left\{g_{i}=x_{1}^{m-i} x_{3}^{i+1}-x_{2}^{m-i+1} x_{4}^{i}, 0 \leq i \leq m, f_{j}^{h}=t x_{3}^{j} x_{4}^{m-j}-x_{1}^{j+1} x_{2}^{m-j} 0 \leq j \leq\right.$ $\left.m, h=x_{1} x_{4}-x_{2} x_{3}\right\}$.

Lemma 3.6. $G^{h}$ is a Gröbner basis with respect to the lexicographic order with $t>x_{4}>x_{2}>x_{3}>x_{1}$.

Proof. $S\left(g_{i}, g_{j}\right), S\left(g_{i}, h\right)$ and $S\left(f_{i}^{h}, f_{j}^{h}\right)=S\left(f_{i}, f_{j}\right) \rightarrow_{G^{h}} 0$ from Lemma 3.5] $S\left(f_{i}^{h}, g_{j}\right)=x_{1}^{m-j} x_{3}^{i+j+1-m} f_{m}^{h}+x_{1}^{i+1} g_{i+j-m}$ for $j \geq m-i$. For $j<m-i, S\left(f_{i}^{h}, g_{j}\right)=$ $x_{1}^{i+1} x_{2}^{m-i-j} g_{0}+x_{1}^{i+1} x_{3} f_{i+j}^{h}$. Thus, $S\left(f_{i}^{h}, g_{j}\right) \rightarrow_{G^{h}} 0$. For $i \neq m, S\left(f_{i}^{h}, h\right)=x_{2} f_{i+1}^{h}$ and $S\left(f_{i}^{h}, h\right) \rightarrow G^{h} 0$, while $S\left(f_{m}^{h}, h\right) \rightarrow_{G^{h}} 0$, since $\operatorname{gcd}\left(\operatorname{in}\left(f_{m}^{h}\right), i n(h)\right)=1$.

Proof of Proposition 3.4. According to the tangent cone algorithm, we must compute a Gröbner basis from $G^{h}$ with respect to a monomial order, such that among monomials of the same total degree, any monomial involving $t$ is greater than any monomial involving only $x_{1}, \ldots, x_{4}$, which is done in Lemma 3.6 Again from the tangent cone algorithm, $I\left(C_{m}\right)_{*}$ is generated by $\left\{g_{i}=x_{1}^{m-i} x_{3}^{i+1}-x_{2}^{m-i+1} x_{4}^{i}\right.$ with $0 \leq i \leq m, f_{j}^{\prime}=x_{3}^{j} x_{4}^{m-j}$ with $\left.0 \leq j \leq m, h=x_{1} x_{4}-x_{2} x_{3}\right\}$. Since $g_{m}$ can be generated by $f_{0}^{\prime}$ and $f_{m}^{\prime}$, we can give a minimal generator set $G_{*}$ for $I\left(C_{m}\right)_{*}$ such that $G_{*}=\left\{g_{i}=x_{1}^{m-i} x_{3}^{i+1}-x_{2}^{m-i+1} x_{4}^{i} 0 \leq i \leq m-1, f_{j}^{\prime}=x_{3}^{j} x_{4}^{m-j} 0 \leq j \leq m\right.$, $\left.h=x_{1} x_{4}-x_{2} x_{3}\right\}$. 
We can now prove Theorem 3.1 .

Proof of Theorem [3.1. $I\left(C_{m}\right)_{*}$ is generated by $G_{*}$ which is also a minimal Gröbner basis with respect to the reverse lexicographic order with $x_{4}>x_{2}>x_{3}>x_{1}$ $\left(S\left(f_{i}^{\prime}, f_{j}^{\prime}\right)=0, S\left(f_{j}^{\prime}, h\right) \rightarrow_{G_{*}} 0, S\left(g_{i}, h\right) \rightarrow_{G_{*}} 0, S\left(g_{i}, g_{j}\right) \rightarrow_{G_{*}} 0\right.$ and $S\left(f_{i}^{\prime}, g_{j}\right) \rightarrow_{G_{*}}$ $0)$. We can now apply Theorem 2.1. Since $x_{1}$ does not divide $i n\left(g_{i}\right)=x_{2}^{m-i+1} x_{4}^{i} 1 \leq$ $i \leq m, i n\left(f_{j}^{\prime}\right)=x_{3}^{j} x_{4}^{m-j} 0 \leq j \leq m$ and $i n(h)=x_{2} x_{3}, k\left[x_{1}, x_{2}, x_{3}, x_{4}\right] / I\left(C_{m}\right)_{*}$ is Cohen-Macaulay.

Theorem 3.1 shows that the monomial curve $C_{m}$, for which $\mu\left(I\left(C_{m}\right)_{*}\right)=2 m+2$ has Cohen-Macaulay tangent cone. Thus, there are monomial curves with arbitrary large minimal number of generators of $I\left(C_{m}\right)_{*}$ and Cohen-Macaulay tangent cones.

Remark 3.7. It is now trivial to compute the Hilbert series of the associated graded ring of the family of curves $C_{m}$. Since $G=k\left[x_{1}, x_{2}, x_{3}, x_{4}\right] / I\left(C_{m}\right)_{*}$ is CohenMacaulay, $G$ and its Artinian reduction $G /\left(x_{1}\right)$ have the same $h$-polynomial. A direct computation shows that the Hilbert series $H_{m}(t)$ of the associated graded ring of the monomial curve $C_{m}$ is given by

$$
H_{m}(t)=\frac{\sum_{i=0}^{m-1}(2 i+1) t^{i}+m t^{m}}{1-t} .
$$

Remark 3.8. (a) By the same approach, the monomial curves $C_{n}$ having the parameterization

$$
x_{1}=t^{n(n+1)+1}, x_{2}=t^{n(n+1)+2}, x_{3}=t^{(n+1)^{2}+1}, x_{4}=t^{(n+1)^{2}+2}
$$

with $n \geq 3$ can be shown to have Cohen-Macaulay tangent cones and $\mu\left(I\left(C_{n}\right)_{*}\right)=$ $2 n+3$.

(b) By a similar approach, Bresinsky curves [3] $C_{q_{2}}$ (which he used for proving that the defining ideal of the monomial curves in affine $l$-space for $l \geq 4$ may have an arbitrary minimal number of generators) having the parameterization

$$
x_{1}=t^{q_{1} q_{2}}, x_{2}=t^{q_{1} d_{1}}, x_{3}=t^{q_{1} q_{2}+d_{1}}, x_{4}=t^{q_{2} d_{1}}
$$

with $q_{1}=q_{2}+1, q_{2}$ even, $q_{2} \geq 4, d_{1}=q_{2}-1$ can also be shown to have CohenMacaulay tangent cones. The approach depends on checking that $x_{4}$ is not a zero divisor in the associated graded ring by considering the generators $F(\nu, \mu)$, since the homogeneous summands of the least degree of $F(\nu, \mu)$ 's generate the $I\left(C_{q_{2}}\right)_{*}$.

\section{EXTENSION TO HIGHER DIMENSION}

Consider the curves $C_{m}^{[5]}$ in 5 -space having parameterization,

$x_{1}=t^{2 m(m+1)}, x_{2}=t^{2 m(m+1)+2}, x_{3}=t^{2(m+1)^{2}}, x_{4}=t^{2(m+1)^{2}+2} x_{5}=t^{2(m+1)^{2}+1}$.

Proposition 4.1. $I\left(C_{m}^{[5]}\right)$ is generated by $G_{m}^{[5]}=\left\{g_{i}=x_{1}^{m-i} x_{3}^{i+1}-x_{2}^{m-i+1} x_{4}^{i}\right.$ with $0 \leq i \leq m, f_{j}=x_{3}^{j} x_{4}^{m-j}-x_{1}^{j+1} x_{2}^{m-j}$ with $\left.0 \leq j \leq m, h=x_{1} x_{4}-x_{2} x_{3}, x_{5}^{2}-x_{4} x_{3}\right\}$.

To prove this proposition, we first recall the following lemma of Morales:

Lemma 4.2 ([9, Lemma 3.2]). Let $C$ be a curve having parameterization

$$
x_{1}=\varphi_{1}(t), \ldots, x_{l-1}=\varphi_{l-1}(t), x_{l}=t^{a} .
$$


Let $\beta$ be a positive integer such that $\operatorname{gcd}(a, \beta)=1$, and let $\tilde{C}$ be a curve having parameterization

$$
x_{1}=\varphi_{1}\left(t^{\beta}\right), \ldots, x_{l-1}=\varphi_{l-1}\left(t^{\beta}\right), x_{l}=t^{a} .
$$

For any $f\left(x_{1}, \ldots, x_{l}\right) \in k\left[x_{1}, \ldots, x_{l}\right]$, we denote by $\tilde{f}$ the element $f\left(x_{1}, \ldots, x_{l-1}, x_{l}^{\beta}\right)$. Then if $f_{1}, \ldots, f_{s}$ is a set of generators for $I(C)$, then $\tilde{f}_{1}, \ldots, \tilde{f}_{s}$ is a set of generators for $I(\tilde{C})$.

Proof of Proposition 4.1. Consider the curve $C^{\prime}$,

$$
x_{1}=t^{m(m+1)}, x_{2}=t^{m(m+1)+1}, x_{3}=t^{(m+1)^{2}}, x_{4}=t^{(m+1)^{2}+1}, x_{5}=t^{2(m+1)^{2}+1}
$$

where $x_{5}=x_{3} x_{4}$. Let $f \in I\left(C^{\prime}\right)$; then

$$
\begin{aligned}
f\left(x_{1}, x_{2}, x_{3}, x_{4}, x_{5}\right) & =f\left(x_{1}, x_{2}, x_{3}, x_{4}, x_{5}-x_{3} x_{4}+x_{3} x_{4}\right) \\
& =\left(x_{5}-x_{3} x_{4}\right) f_{1}\left(x_{1}, x_{2}, x_{3}, x_{4}, x_{5}\right)+f_{2}\left(x_{1}, x_{2}, x_{3}, x_{4}\right)=0
\end{aligned}
$$

for $x_{1}=t^{m(m+1)}, x_{2}=t^{m(m+1)+1}, x_{3}=t^{(m+1)^{2}}, x_{4}=t^{(m+1)^{2}+1}, x_{5}=t^{2(m+1)^{2}+1}$, which shows that $f_{2}\left(t^{m(m+1)}, t^{m(m+1)+1}, t^{(m+1)^{2}}, t^{(m+1)^{2}+1}\right)=0$, that is, $f_{2} \in$ $I\left(C_{m}\right)$. Thus, $I\left(C^{\prime}\right)$ is generated by the generator set $G \cup\left\{x_{5}-x_{3} x_{4}\right\}$, where $G$ is the generator set in Proposition 3.2 .

Applying Lemma 4.2 with $C=C^{\prime}$ in (4.4), $\tilde{C}=C_{m}^{[5]}$ in (4.1) and $\beta=2, I\left(C_{m}^{[5]}\right)$ is generated by $G_{m}^{[5]}=G \cup\left\{x_{5}^{2}-x_{3} x_{4}\right\}$.

The generator basis $G_{m}^{[5]}$ is a Gröbner basis with respect to the graded lexicographic order with $x_{5}>x_{4}>x_{2}>x_{3}>x_{1}$, because $G^{\prime}=G \cup\left\{x_{5}^{2}-x_{3} x_{4}\right\}$, $G$ is a Gröbner basis with respect to the graded lexicographic order with $x_{4}>$ $x_{2}>x_{3}>x_{1}$ and the greatest common divisor of leading monomial of any element in $G$ and $x_{5}^{2}$ is 1 . Also, by the same approach the homogenization of the elements of $G_{m}^{[5]}$ by $t$ is a Gröbner basis with respect to the lexicographic order with $t>x_{5}>x_{4}>x_{2}>x_{3}>x_{1}$. Thus, we obtain the tangent cone as in Section 4, and conclude that $C_{m}^{[5]}$ is a monomial curve with $\mu\left(I\left(C_{m}^{[5]}\right)_{*}\right)=2 m+3$ and has a Cohen-Macaulay tangent cone. It is obvious that, with the same method, we can extend the result to all higher dimensions, such that if $C_{m}^{[l]}$ has the parameterization

$$
x_{1}=t^{a_{1}}, \ldots x_{l-1}=t^{a_{l-1}}, x_{l}=t^{a_{l}},
$$

then $C_{m}^{[l+1]}$ is given by

$$
x_{1}=t^{2 a_{1}}, \ldots x_{l-1}=t^{2 a_{l-1}}, x_{l}=t^{2 a_{l}}, x_{l+1}=t^{a_{l-1}+a_{l}} .
$$

Remark 4.3. In an affine $l$-space with $l>4, I\left(C_{m}^{[l]}\right)$ is generated by

$G_{m}^{[l]}=\left\{g_{i}=x_{1}^{m-i} x_{3}^{i+1}-x_{2}^{m-i+1} x_{4}^{i}\right.$ with $0 \leq i \leq m, f_{j}=x_{3}^{j} x_{4}^{m-j}-x_{1}^{j+1} x_{2}^{m-j}$ with $\left.0 \leq j \leq m, h=x_{1} x_{4}-x_{2} x_{3}, x_{5}^{2}-x_{4} x_{3}, \ldots, x_{l}^{2}-x_{l-1} x_{l-2}\right\}$

and $I\left(C_{m}^{[l]}\right)_{*}$ is generated by

$$
\begin{aligned}
G_{m_{*}}^{[l]}= & \left\{g_{i}=x_{1}^{m-i} x_{3}^{i+1}-x_{2}^{m-i+1} x_{4}^{i} \text { with } 0 \leq i \leq m-1, f_{j}^{\prime}=x_{3}^{j} x_{4}^{m-j}\right. \text { with } \\
& \left.0 \leq j \leq m, h=x_{1} x_{4}-x_{2} x_{3}, x_{5}^{2}-x_{4} x_{3}, \ldots, x_{l}^{2}-x_{l-1} x_{l-2}\right\}
\end{aligned}
$$

which is also a minimal Gröbner basis with respect to the reverse lexicographic order with $x_{l}>x_{l-1}>\ldots>x_{5}>x_{4}>x_{2}>x_{3}>x_{1}$ from the construction. 
Thus from Theorem 2.1] $C_{m}^{[l]}$ has Cohen-Macaulay tangent cone at the origin. Also, $\mu\left(I\left(C_{m}^{[l]}\right)_{*}\right)=2 m+l-2$.

Remark 4.4. We can compute the Hilbert series of $G=k\left[x_{1}, \ldots, x_{l}\right] / I\left(C_{m}^{[l]}\right)_{*}$. Since $G_{m_{*}}^{[l]}$ is a Gröbner basis with respect to the reverse lexicographic order with $x_{l}>$ $x_{l-1}>\ldots>x_{5}>x_{4}>x_{2}>x_{3}>x_{1}, k\left[x_{1}, \ldots, x_{l}\right] / I\left(C_{m}^{[l]}\right)_{*}$ and $k\left[x_{1}, \ldots, x_{l}\right] / \operatorname{in}\left(G_{m_{*}}^{[l]}\right)$ have the same Hilbert series, where $\operatorname{in}\left(G_{m_{*}}^{[l]}\right)$ is the ideal generated by the leading terms of the elements of the generator set $G_{m_{*}}^{[l]}$ with respect to this order. By using [1. Proposition 2.4] and Remark 3.7 the Hilbert series $H_{m}^{[l]}(t)$ of the associated graded ring of the monomial curve $C_{m}^{[l]}$ for $l \geq 4$ is given by

$$
H_{m}^{[l]}(t)=\frac{(1+t)^{l-2}\left(\sum_{i=0}^{m-1}(2 i+1) t^{i}+m t^{m}\right)}{1-t} .
$$

As a result, in every affine $l$-space with $l \geq 4$, there are monomial curves having Cohen-Macaulay tangent cone with arbitrarily large minimal number of generators.

\section{ACKNOWLEDGEMENTS}

I would like to thank W. Vasconcelos and Rutgers University Department of Mathematics for their generous support and hospitality during my visit. I would also like to thank S. Sertöz, W. Vasconcelos and G. Valla for helpful conversations and their comments.

\section{REFERENCES}

1. Bayer, D., Stillman, M., Computation of Hilbert functions, J. Symbolic Computation 14 (1992), 31-50. MR 94f:13018

2. D. Bayer and M. Stillman, Macaulay, A system for computation in algebraic geometry and commutative algebra, 1992, available via anonymous ftp from math.harvard.edu.

3. Bresinsky, H., On prime ideals with generic zero $x_{i}=t^{n_{i}}$, Proc. Amer. Math. Soc. 47, No.2 (1975), 329-332. MR 52:10741

4. Cavaliere, M. P., Niesi, G., On form ring of a one-dimensional semigroup ring, Lecture Notes in Pure and Appl. Math. 84 (1983), 39-48. MR 84i:13018

5. Cox, D., Little, J., O'Shea, D., Ideals, varieties and algorithms, Springer-Verlag, 1992. MR 93j:13031

6. Garcia, A., Cohen-Macaulayness of the associated graded of a semigroup ring, Comm. in Algebra 10, No.4 (1982), 393-415. MR 83k:13013

7. Herzog, J., Generators and relations of abelian semigroups and semigroup rings, Manuscripta Math. 3 (1970), 175-193. MR 42:4657

8. Herzog, J., When is a regular sequence super regular?, Nagoya Math. J., 83 (1981), 183-195. MR 83c:13009

9. Morales, M., Noetherian symbolic blow-ups, Journal of Algebra 140 (1991), 12-25. MR 92c: 13020

10. Robbiano, L., Valla, G., On the equations defining tangent cones, Math. Proc. Camb. Phil. Soc. 88 (1980), 281-297. MR 81i:14004

11. Sally, J., Good embedding dimensions for Gorenstein singularities, Math. Ann. 249 (1980), 95-106. MR 82c:13031

12. Vasconcelos, W. V., Computational methods in commutative algebra and algebraic geometry, Springer-Verlag, 1998. MR 99c:13048

Department of Mathematics, Bilkent University, Ankara, Turkey 06533

E-mail address: sarslan@fen.bilkent.edu.tr

Current address: Department of Mathematics, METU, Ankara, Turkey 06531

E-mail address: feza@arf.math.metu.edu.tr 